\title{
Introducing Metabolic Messengers
}

This month, we introduce a new series of articles, called Metabolic Messengers, dedicated to molecules involved in cellular communication and inter-tissue cross-talk.

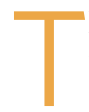

he capability to communicate the metabolic state of a cell to neighbouring cells and tissues is crucial for maintaining cellular and organismal energy homeostasis. Unsurprisingly, a wide variety of mechanisms exist to sense, distribute and integrate such information, often involving signals or molecules that travel between cells or to distant organs. Unravelling the complex, integrated nature of such cross-talk is a key challenge in metabolic physiology. This month, we introduce a new series of articles, called Metabolic Messengers, dedicated to molecules involved in cellular communication and inter-tissue cross-talk. The first article in the series, on adiponectin, can be found in this issue and will give you a taste of what is to come.

The Metabolic Messengers series is unique in that it comprises concise, focused articles that provide an authoritative, high-level overview of the history, physiology and regulation of metabolically relevant molecules. At a time when the number of research articles and reviews is constantly increasing, we feel that this short article format is valuable because it provides a brief yet comprehensive overview of important metabolic and homeostatic mediators. We envision this series to serve both as an introduction for scientists who are new to the field and as a brief refresher for more experienced researchers.

Published occasionally every few months, Metabolic Messengers will cover 'classical' hormones as well as mediators of inter-tissue cross-talk, cell-cell communication and even metabolic signalling within cells. All articles will be connected by their look and feel, featuring recurring display items, such as a timeline detailing key discoveries or an overview of target sites, as appropriate. We look forward to seeing the series evolve over time as new messengers are discovered, and we hope that you will find the Metabolic Messengers useful for study and research.

Metabolic messengers and communication at different biological levels will also be the topic of an upcoming conference organized by Instituto Serrapilheira, Nature Metabolism and Nature Cell Biology, from 15-18 October 2019 in Rio de Janeiro, Brazil. The 'Advances in Metabolic Communication' conference will bring together a diverse group of experts to discuss the latest developments in metabolic cross-talk-among organelles, cells and tissues-and on the role of miscommunication in disease. More information about speakers and the program can be found on the conference website (https://www.nature.com/natureconferences/ amc19/index.html). We hope to meet many of you in Rio de Janeiro later this year!

Published online: 14 March 2019 https://doi.org/10.1038/s42255-019-0049-4 